\title{
Quantum tomography via Non-orthogonal basis and weak values
}

\author{
J.J. Díaz and I. Sainz \\ Dept. de Física, Universidad de Guadalajara, Revolución 1500, 44420 Guadalajara, Jal., México. \\ A. B. Klimov \\ Dept. de Física, Universidad de Guadalajara, Revolución 1500, 44420 Guadalajara, Jal., México. and \\ Center for Optics and Photonics, Universidad de Concepción Casilla 4016, Concepción, Chile
}

(Dated: October 6, 2018)

\begin{abstract}
Using a relation between a bi-orthogonal set of equiseparable bases and the weak values of the density matrix we derive an explicit formula for its tomographic reconstruction completely analogous to the standard mutually unbiased bases expansion. With the simple example of a qubit is evidenced the relationship between weak values, measured probabilities and the separation between non-orthogonal bases.
\end{abstract}

\section{INTRODUCTION}

A classical state is an observable that can be completely determined through simultaneous measurements of a certain set of (conjugate) physical variables. On the other hand, quantum states are not observables and the conjugate variables corresponding to non-commuting operators cannot be measured simultaneously. The complete description of a quantum system implies a reconstruction of a given quantum state: the so-called Quantum State Tomography (QST) [1] procedure. The general idea for QST of a finite-dimensional system, of dimension $p$, is a simultaneous measurement of $p-1$ probabilities in each of $p+1$ linearly independent bases in order to obtain the $p^{2}-1$ real parameters describing the density matrix of the system. It has been proved [2] that (orthogonal) Mutually Unbiased Bases (MUB) constitute the optimal measurement set in the case of a priori unknown quantum state, since the informational redundancy among different measurements is removed. MUB tomography schemes were successfully employed in recent experiments $\underline{3}, 4]$.

The situation becomes more involved if the measurement space (i.e. set of the bases where a quantum state can be projected out) is restricted [5]. In this case, a set of non-orthogonal bases can be used for the density matrix reconstruction. A scheme, which preserves most of the standard MUB tomography features [6], is based on equidistant non-orthogonal bases [7], and characterized by a separation parameter $\lambda(\lambda=0$ corresponds to the orthogonal case). In the frame of this approach the density matrix is expanded in terms of the projectors over specific (non-orthogonal) bases and the expansion coefficients are the probabilities measured in the corresponding biorthogonal bases. An alternative reconstruction method involving non-orthogonal measurements (within the positive operator valued measure approach) was discussed in [8].

Unfortunately, the above mentioned non-orthogonal tomographic schemes have a drawback: the probabilities associated to every independent element are scaled by a factor $\sim(1-\lambda)^{-1}$, so that when the basis elements are close it becomes singular. This singularity is apparent in the sense that it disappears if exact probalitities are substituted in the reconstruction equation. However, since (exprimentally) the tomography is performed with estimated probabilities, the statistical errors lead to large deviations from the real density matrix as $\lambda \rightarrow 1$, even in the case of perfect measurements.

Algebraically, non-orthogonal basis vectors are eigenstates of some non-Hermitian operators. Thus, tomography in non-orthogonal bases can be associated with measurements of non-Hermitian operators [9, 10], which naturally appear in reconstruction schemes via weak measurements [9, 11-14] (for experimental implementation see Ref. [9, 13]). So we find that, non-orthogonal tomography should be connected to the concept of weak measurements and the two-vector formalism (assignment of pre-and-post-selection ensembles) [15, 16], that combined lead to the notion of the weak value of an observable 17 (see [18] and references therein). Numerous applications of weak values in foundations of quantum mechanics [11, 15, 17, 19 21], superluminal light propagations [22] and quantum metrology [23] have attracted considerable attention in recent years. On the other hand, since weak measurements do not "completely" disturb the quantum state (in the sense of wave function collapse), they seem to be naturally related to projections onto mutually non-orthogonal states.

Here, we propose a quantum tomography scheme for finite-dimensional systems of prime dimensions with equiseparable (non-othogonal) bases [7], 6] using the advantage of employing a complete set of bi-orthogonal MUBs. We present an explicit reconstruction expression for the density matrix, which is an analogy to the standard (orthogonal) MUB expansion, except that it is spanned by the bi-orthogonal projectors and the role of the measured probabilities is played by the weak values of the density matrix. On a single qubit example we show how the weak values and the measured probabilities are related in terms of rotations in the Bloch sphere. 


\section{EQUIDISTANT NON-ORTHOGONAL BASES}

In this Section we briefly summarize previous results obtained on the non-orthogonal bases [7], [24] and bi-orthogonal MUBs [6, 25]. Given a quantum system of dimension $p$, where $p \geq 3$ is a prime number $(p=2$ is discussed below), a set of $p$ non-orthogonal equidistant bases $\left\{\left|\psi_{m}^{s}\right\rangle, m=0, \ldots, p-1\right\}$, where the super-index $s=1, \ldots, p$ labels the bases, can be constructed. The equidistant condition reads as

$$
\left|\left\langle\psi_{m}^{s} \mid \psi_{n}^{s}\right\rangle\right|=(1-\lambda) \delta_{m n}+\lambda,
$$

where $-(p-1)^{-1} \leq \lambda \leq 1$ is the separation parameter between elements of the same basis. It is remarkable that a set of $p$ bi-orthogonally unbiased equidistant bases $\left\{\left|\phi_{m}^{s}\right\rangle, s=1, \ldots, p\right\}$, with separation $\eta=-\lambda /[1+(p-2) \lambda]$, can be found, where the unbiasedness condition reads as

$$
\left|\left\langle\phi_{m}^{t} \mid \psi_{n}^{s}\right\rangle\right|^{2}=\frac{\delta_{s t} \delta_{n m}}{\mu}+\frac{1-\delta_{s t}}{\mu p},
$$

with

$$
\mu=\frac{1+(p-2) \lambda}{(1-\lambda)(1+(p-1) \lambda)}
$$

In particular, one has $\left\langle\phi_{m}^{s} \mid \psi_{n}^{s}\right\rangle=\delta_{n m} / \sqrt{\mu}$.

The elements of the basis $\left\{\left|\psi_{m}^{p}\right\rangle\right\}$ can be considered as eigenstates of a non-unitary cyclic operator $Z$, so that $Z^{t}\left|\psi_{m}^{p}\right\rangle=\omega^{t m}\left|\psi_{m}^{p}\right\rangle$, where $\omega=e^{2 i \pi / p}, Z^{p}=\mathrm{I}$, hence

$$
Z^{t}=\sum_{k=0}^{p-1} \omega^{t k} \tilde{P}_{k}^{p}
$$

where we have introduced the bi-orthogonal normalized projectors $\tilde{P}_{k}^{s}=\sqrt{\mu}\left|\psi_{k}^{s}\right\rangle\left\langle\phi_{k}^{s}\right|, s=1, \ldots, p$ satisfying the following decomposition of identity [24, 25],

$$
\mathrm{I}=\sum_{k=0}^{p-1} \tilde{P}_{k}^{s}
$$

and all the operations are done $\bmod p$.

The elements of the $s$-th basis, for $s \neq p$, are eigenstates of the set of non-unitary cyclic operators which spectral bi-orthogonal decomposition is given by

$$
Z^{t} X^{r}=\sum_{k=0}^{p-1} \omega^{\left(2^{-1} t-k\right) r} \tilde{P}_{k}^{s}
$$

where $s=t r^{-1}, r, t=1, \ldots p-1$, and the unitary, cyclic, shift operators are defined by

$$
X^{r}=\sqrt{\mu} \sum_{k=0}^{p-1}\left|\psi_{k+r}^{p}\right\rangle\left\langle\phi_{k}^{p}\right| .
$$

The bi-orthogonal bases are eigenstates of the correspondent adjoint operators [24].

The last, $p+1$-th basis required for the tomographic expansion is orthonormal and its elements are the eigenstates of the unitary operators $X^{r}$. From now on, we will label this basis with the upper index 0:

$$
X^{r}\left|\psi_{m}^{0}\right\rangle=\omega^{-r m}\left|\psi_{m}^{0}\right\rangle
$$

Explicit relations between all these bases can be consulted in Ref. [25]. In particular, the overlap between the element $\left|\psi_{0}^{0}\right\rangle$ and the bases $\left\{\left|\psi_{m}^{s}\right\rangle\right\},\left\{\left|\phi_{m}^{s}\right\rangle\right\}$ has a constant absolute value,

$$
\begin{aligned}
\left|\left\langle\psi_{0}^{0} \mid \psi_{m}^{s}\right\rangle\right|^{2} & =\frac{1+(p-1) \lambda}{p}, \\
\left|\left\langle\psi_{0}^{0} \mid \phi_{m}^{s}\right\rangle\right|^{2} & =\frac{1+(p-1) \eta}{p},
\end{aligned}
$$


while for the rest of elements $\left|\psi_{n}^{0}\right\rangle, n \neq 0$, the overlap is given by

$$
\left|\left\langle\psi_{n}^{0} \mid \psi_{m}^{s}\right\rangle\right|^{2}=\frac{1-\lambda}{p}, \quad\left|\left\langle\psi_{n}^{0} \mid \phi_{m}^{s}\right\rangle\right|^{2}=\frac{1-\eta}{p} .
$$

Therefore, $\left|\psi_{0}^{0}\right\rangle$ has a very specific property: all the components of the equidistant bases $\left\{\left|\psi_{k}^{s}\right\rangle, s=1, \ldots, p\right\}$ approach to $\left|\psi_{0}^{0}\right\rangle$ in the near parallel limit, $\lambda \rightarrow 1$; while the bi-orthogonal bases are concentrated in the hyperplane orthogonal to this state.

\section{WEAK VALUES AND QUANTUM TOMOGRAPHY}

The monomials $\left\{Z^{t} X^{r}\right\}$, for $t, r=0, \ldots, p-1$ (where $t=r=0$ corresponds to the identity operator), form a complete operational basis that allows to reconstruct an arbitrary operator acting in the $p$ dimensional Hilbert space. In particular, the density matrix $\hat{\rho}$ can be expanded as follows

$$
\hat{\rho}=\sum_{t, r=0}^{p-1} c_{t r} Z^{t} X^{r}
$$

were the coefficients $c_{t r}$ are given in terms of the expectation values of the non-Hermitian operators $\left\{X^{r \dagger} Z^{-t}\right\}$ :

$$
c_{t r}=\frac{1}{p} \operatorname{Tr}\left[\hat{\rho} X^{r \dagger} Z^{-t}\right]=\frac{\left\langle X^{r \dagger} Z^{-t}\right\rangle}{p},
$$

The sets of operators $\left\{Z^{t} X^{r}\right\}$, and $\left\{X^{r \dagger} Z^{-t}\right\}$ are reciprocal [20],

$$
\begin{aligned}
\operatorname{Tr}\left\{X^{r} X^{r^{\prime} \dagger}\right\} & =p \delta_{r, r^{\prime}}, \\
\operatorname{Tr}\left\{Z^{t} Z^{-t^{\prime}}\right\} & =p \delta_{t, t^{\prime}}
\end{aligned}
$$

which grants the reconstruction Eq. (10).

The expansion coefficients $c_{t r}$ have a peculiar interpretation. First of all, $c_{00}=1 / p$ due to the normalization condition; the coefficient of the unitary terms, $X^{r}$ are

$$
c_{0 r}=\frac{1}{p} \sum_{k=0}^{p-1} \omega^{-k r} p_{0 k}
$$

where $p_{0 k}=\left\langle\psi_{k}^{0}|\hat{\rho}| \psi_{k}^{0}\right\rangle$ is the probability of detecting elements of the orthonormal basis $\left\{\left|\psi_{k}^{0}\right\rangle\right\}$;

The coefficients of the non-unitary elements in the expansion Eq. (10) have the following form,

$$
\begin{aligned}
c_{t 0} & =\frac{1}{p} \sum_{k=0}^{p-1} \omega^{-t k} W_{k}^{p}(\rho), \\
c_{t r} & =\frac{\omega^{2^{-1}} t r}{p} \sum_{k=0}^{p-1} \omega^{r k} W_{k}^{t r^{-1}}(\rho), \quad r \neq 0,
\end{aligned}
$$

where

$$
W_{k}^{s}(\rho)=\frac{\left\langle\phi_{k}^{s}|\hat{\rho}| \psi_{k}^{s}\right\rangle}{\left\langle\phi_{k}^{s} \mid \psi_{k}^{s}\right\rangle}, \quad s=1, \ldots, p,
$$

is the weak value of the density matrix for the initial state $\left|\psi_{k}^{s}\right\rangle$ and post-selected state $\left|\phi_{k}^{s}\right\rangle$ (in what follows we will write $W_{k}^{s}$ for short). The concept of the weak value of the density matrix Eq. (14) in a non-normalized form (usually called the Dirac or Kirkwood distribution) has been used in the context of quantum state tomography via weak measurements [9, 11 14, 16, 20]. It was also argued in [20, 21] that such a weak value is a complex number corresponding to the joint probability of measurements that cannot be done simultaneously, i.e. projection into $\left|\psi_{k}^{s}\right\rangle\left\langle\psi_{k}^{s}\right|$ and $\left|\phi_{k}^{s}\right\rangle\left\langle\phi_{k}^{s}\right|$. 
Substituting the spectral decomposition of monomials $Z^{t} X^{r}$ into Eq. (10) we arrive to the following reconstruction equation

$$
\hat{\rho}=\sum_{k=0}^{p-1} p_{0 k} P_{k}^{0}+\sum_{s=1}^{p} \sum_{k=0}^{p-1} W_{k}^{s} \tilde{P}_{k}^{s}-\mathrm{I},
$$

where $P_{k}^{0}=\left|\psi_{k}^{0}\right\rangle\left\langle\psi_{k}^{0}\right|$ are the projectors onto the orthogonal basis Eq.(77). Eq.(15) is our main result an it exhibits how the weak values of the density matrix naturally appear in the frame of the bi-orthogonal MUB tomography. It should be stressed that the above reconstruction equation does not contain any divergent factors (in the limit $\lambda \rightarrow 1$ ) proper for non orthogonal projective tomography [ $[$,, 8$]$. In the orthogonal limit, $\lambda=0$, the weak values become true probabilities, and the $\tilde{P}_{k}^{s}$ are transformed into orthogonal projectors so that the standard MUB reconstruction expression 2] is recovered. It can be easily shown that if the density matrix expansion Eq.(15) is performed on $\tilde{P}_{k}^{s \dagger}$ instead of $\tilde{P}_{k}^{s}$, then the corresponding coefficients are $W_{k}^{s *}$, i.e.

$$
\hat{\rho}=\sum_{k=0}^{p-1} p_{0 k} P_{k}^{0}+\sum_{s=1}^{p} \sum_{k=0}^{p-1} W_{k}^{s *} \tilde{P}_{k}^{s \dagger}-\mathrm{I} .
$$

The above equations ensure the hermiticity of the expansions Eqs.(15)-(16).

It is worth noting that the weak values Eq.(14) satisfy the normalization condition

$$
\sum_{k=0}^{p-1} W_{k}^{s}=1
$$

in a complete analogy with the sum of probabilities $\sum_{k=0}^{p-1} p_{0 k}=1$ measured in the orthogonal basis $\left\{\left|\psi_{k}^{0}\right\rangle\right\}$.

Formally, the reconstruction equation (15) depends on $(2 p+1)(p-1)$ real parameters since the weak values $W_{k}^{s}$ are complex numbers. However, such redundancy is only apparent due to specific relations between the weak values and their complex conjugates. These relations are straightforward to obtain by equalling matrix elements of Eq. (15) and Eq.(16) in the orthogonal basis $\left\{\left|\psi_{k}^{0}\right\rangle\right\}$ :

$$
\begin{array}{r}
\sum_{k=0}^{p-1} \omega^{-(n-m) k}\left[2 i\left(1-\lambda \delta_{m 0}\right) \operatorname{Im} W_{k}^{p}-p \lambda \delta_{m 0} W_{k}^{p *}\right]= \\
\sum_{s=1}^{p-1} \sum_{k=0}^{p-1} \omega^{(2 s)^{-1}(n-m)[2 k-(n+m)]}\left[2 i\left(1-\lambda \delta_{m 0}\right) \operatorname{Im} W_{k}^{s}-p \lambda \delta_{m 0} W_{k}^{s *}\right]
\end{array}
$$

where $m=0, \ldots, p-2, n=1, \ldots, p-1$ and $n>m$. There are $p(p-1) / 2$ complex conditions, so that the total number of real parameters required for the reconstruction in Eq.(15) is reduced from $(2 p+1)(p-1)$ to the $p^{2}-1$ as it should be.

\section{DIMENSION TWO}

The general expansion Eq.(15) is not valid in the special case of dimension two (the quantity $2^{-1}$ is undefined). In this Section we present explicit reconstruction equations for $p=2$ and discuss the posiibility of their experimental implementation. Let us consider a basis constituted by two states $\left\{\left|\psi_{0}^{2}\right\rangle,\left|\psi_{1}^{2}\right\rangle\right\}$ with the overlap condition $\left\langle\psi_{0}^{2} \mid \psi_{1}^{2}\right\rangle=\lambda$, the corresponding (normalized) bi-orthogonal basis is defined by

$$
\begin{aligned}
& \left|\phi_{0}^{2}\right\rangle=\frac{1}{\sqrt{1-\lambda^{2}}}\left(\left|\psi_{0}^{2}\right\rangle-\lambda\left|\psi_{1}^{2}\right\rangle\right), \\
& \left|\phi_{1}^{2}\right\rangle=\frac{1}{\sqrt{1-\lambda^{2}}}\left(\left|\psi_{1}^{2}\right\rangle-\lambda\left|\psi_{0}^{2}\right\rangle\right),
\end{aligned}
$$

where $\mu=\left(1-\lambda^{2}\right)^{-1}$, and $\left\langle\phi_{0}^{2} \mid \phi_{1}^{2}\right\rangle=-\lambda$. The cyclic non-unitary operator $Z$ is then given by the bi-orthogonal spectral decomposition

$$
Z=\frac{1}{\sqrt{1-\lambda^{2}}}\left(\left|\psi_{0}^{2}\right\rangle\left\langle\phi_{0}^{2}|-| \psi_{1}^{2}\right\rangle\left\langle\phi_{1}^{2}\right|\right)
$$


The unitary shift operator $X$ has the form

$$
X=\frac{1}{\sqrt{1-\lambda^{2}}}\left(\left|\psi_{0}^{2}\right\rangle\left\langle\phi_{1}^{2}|+| \psi_{1}^{2}\right\rangle\left\langle\phi_{0}^{2}\right|\right),
$$

and their eigenstates $\left|\psi_{0,1}^{0}\right\rangle=\left(\left|\psi_{0}^{2}\right\rangle \pm\left|\psi_{1}^{2}\right\rangle\right) / \sqrt{2(1 \pm \lambda)}$ are orthonormal.

A bi-orthogonal unbiased to $\left\{\left|\phi_{0,1}^{2}\right\rangle\right\}$ basis $\left|\psi_{0,1}^{1}\right\rangle=\left(\left|\psi_{0}^{2}\right\rangle \pm i\left|\psi_{1}^{2}\right\rangle\right) / \sqrt{2},\left\langle\psi_{0}^{1} \mid \psi_{1}^{1}\right\rangle=1$ is formed by eigenstates of the operator $Z X$, which spectral decomposition is

$$
Z X=\frac{i}{\sqrt{1-\lambda^{2}}}\left(\left|\psi_{0}^{1}\right\rangle\left\langle\phi_{0}^{1}|-| \psi_{1}^{1}\right\rangle\left\langle\phi_{1}^{1}\right|\right) .
$$

The operators $X, Z, Z X$ and the identity form a complete set of linearly independent operators, so that the density matrix can be expanded as

$$
\hat{\rho}=\frac{\mathrm{I}}{2}+c_{01} X+c_{10} Z+c_{11} Z X
$$

In this particular case $Z^{-1}=Z$, and the expansion coefficients are given by

$$
\begin{aligned}
c_{01} & =\frac{1}{2} \operatorname{Tr}\left(\hat{\rho} X^{\dagger}\right)=\frac{1}{2}\left(p_{00}-p_{01}\right), \\
c_{10} & =\frac{1}{2} \operatorname{Tr}(\hat{\rho} Z)=\frac{1}{2}\left(W_{0}^{2}-W_{1}^{2}\right), \\
c_{11} & =\frac{1}{2} \operatorname{Tr}\left(\hat{\rho} X^{\dagger} Z\right)=\frac{i}{2}\left(W_{1}^{1}-W_{0}^{1}\right),
\end{aligned}
$$

where the probabilities $p_{0 k}$ and the weak values $W_{k}^{s}$ are defined as in the previous Section. Using the spectral decomposition of operators $X, Z, Z X$ and the completeness relations $p_{00}+p_{01}=W_{0}^{s}+W_{1}^{s}=1$ for $s=1,2$, the reconstruction equation for the density matrix is given by Eq.(15). There is a single (complex) condition imposed on $W_{0}^{2}$ and $W_{0}^{1}$,

$$
\begin{aligned}
& (1+\lambda)\left[W_{0}^{2 *}+i W_{0}^{1 *}\right] \\
= & (1-\lambda)\left[W_{0}^{2}+i W_{0}^{1}\right]+\lambda(1+i) .
\end{aligned}
$$

There is a simple scheme for obtaining the weak values $W_{k}^{1,2}, k=0,1$ requiered for the reconstruction Eq. (18).

First, let us choose a Pauli matrix as a unitary operator: $X=\sigma_{z}=\left|\psi_{0}^{0}\right\rangle\left\langle\psi_{0}^{0}|-| \psi_{1}^{0}\right\rangle\left\langle\psi_{1}^{0}\right|$, so that the probabilities involved in the coefficient $c_{01}$ are $p_{0 k}=\left\langle\psi_{k}^{0}|\hat{\rho}| \psi_{k}^{0}\right\rangle, k=0,1$. If we define the observables

$$
\hat{M}_{k}^{y}(\alpha)=R_{y}\left((-1)^{k} \alpha\right) \sigma_{z} R_{y}^{\dagger}\left((-1)^{k} \alpha\right),
$$

for $k=0,1$, and $R_{y}(\alpha)=\exp \left(i \alpha \sigma_{y} / 2\right)$, which are rotations of $\sigma_{z}$ around the $y$ axis, such that $\lambda=\cos \alpha / 2$, then, the weak values $W_{k}^{2}$ are obtained by post-selecting the states $\left|\psi_{k}^{2}\right\rangle$ after the weak measurement of the observables $\hat{M}_{k}^{y}(\alpha)$

$$
W_{k}^{2}=\frac{\mu}{2}-\frac{\mu}{2}\left\langle\psi_{k}^{2}\left|\hat{M}_{k}^{y}(\alpha) \hat{\rho}\right| \psi_{k}^{2}\right\rangle .
$$

Observe, that the states $\left|\psi_{k}^{2}\right\rangle$ can be obtained by rotating $\left|\psi_{0}^{0}\right\rangle$ in the direction opposite that used in Eq.(20): $\left|\psi_{k}^{2}\right\rangle=R_{y}^{\dagger}\left((-1)^{k} \alpha\right)\left|\psi_{0}^{0}\right\rangle$.

Similarly, $W_{k}^{1}, k=0,1$ can be accessed by post-selecting the states $\left|\psi_{k}^{1}\right\rangle=R_{x}^{\dagger}\left((-1)^{k} \alpha\right)\left|\psi_{0}^{0}\right\rangle$ after the weak measurement of the observable

$$
\hat{M}_{k}^{x}(\alpha)=R_{x}\left((-1)^{k} \alpha\right) \sigma_{z} R_{x}^{\dagger}\left((-1)^{k} \alpha\right)
$$

where $R_{x}(\alpha)=\exp \left(i \alpha \sigma_{x} / 2\right)$ :

$$
W_{k}^{1}=\frac{\mu}{2}-\frac{\mu}{2}\left\langle\psi_{k}^{1}\left|\hat{M}_{k}^{x}(\alpha) \hat{\rho}\right| \psi_{k}^{1}\right\rangle .
$$

Nevertheless, it follows from the completeness relation $W_{0}^{s}+W_{1}^{s}=1$, for $s=1,2$ that only the observable $\hat{M}_{0}^{y}(\alpha)$ (or $\left.\hat{M}_{1}^{y}(\alpha)\right)$ is requiered to obtain $W_{0}^{2}, W_{1}^{2}$ (the same happens with $\left.W_{0}^{1}, W_{1}^{1}\right)$. Besides, due to relation Eq. (19) only one 
of the weak values, either $W_{0}^{1}$ or $W_{0}^{2}$ should be determined experimentally. Thus, in this reconstruction protocol only two observables are required for the complete determination of an unknown state: strongly measured $\sigma_{z}$ to obtain $p_{0 k}$, and, for instance, the observable $M_{0}^{y}$ which is weakly measured and post-selected in a corresponding state. Let us remember, that it is required to measure three observebales in the framework of the standard orthogonal MUB tomography.

It is worth discussing the geometrical meaning of the weak values $W_{k}^{s}, k=0,1, s=1,2$. Let us express them in a single equation as follows

$$
W_{k}^{s}=\sqrt{\mu}\left\langle\phi_{k}^{s}|\hat{\rho}| \psi_{k}^{s}\right\rangle=\mu\left\langle\psi_{k}^{s}\left|\left(\left|\phi_{k}^{s}\right\rangle\left\langle\phi_{k}^{s}\right| \hat{\rho}\right)\right| \psi_{k}^{s}\right\rangle .
$$

The above can be interpreted as weak measurements of the operators $\left|\phi_{k}^{s}\right\rangle\left\langle\phi_{k}^{s}\right|$ in the initial state $\hat{\rho}$ and final, postselected, states $\left|\psi_{k}^{s}\right\rangle$, for $k=0,1, s=1,2$. The operators to be weakly measured are obtained by rotations around the $x$ (or $y$ ) axis from the projector on the state $\left|\psi_{1}^{0}\right\rangle$ located in the south pole of the Bloch sphere, for instance,

$$
\left|\phi_{k}^{1}\right\rangle\left\langle\phi_{k}^{1}\left|=R_{x}\left((-1)^{k} \alpha\right)\right| \psi_{1}^{0}\right\rangle\left\langle\psi_{1}^{0}\right| R_{x}^{\dagger}\left((-1)^{k} \alpha\right),
$$

while the corresponding post-selection states are obtained from the state orthogonal to $\left|\psi_{1}^{0}\right\rangle$ (the north pole state) and rotated on the same angle but in opposite direction:

$$
\left|\psi_{k}^{1}\right\rangle=R_{x}^{\dagger}\left((-1)^{k} \alpha\right)\left|\psi_{0}^{0}\right\rangle
$$

The rotation angle are determined by $\cos \alpha / 2=\lambda$ so that when $\alpha=\pi(\lambda=0)$ the measured observables coincide with the post-selection projectors and the weak values become the standard projection probabilities on the axes $x$ (or $y$ ), putting in evidence the connection between the measured probabilities and the weak values in the frame of the optimal tomographic reconstruction.

In summary, we have found an explicit relation between the expectation values of a set of specific non-Hermitian operators Eq.(11) and the week values of the density matrix (the normalized Dirac distribution). Such relation allowed us to introduce a tomographic expansion of the density matrix, Eq.(15) in terms of such week values in a form analogous to the standard MUB expansion [2] but free of artificial singularities.

[1] U. Leonhardt, Measuring the quantum state of light (Cambridge University Press, 1997); M. G. A. Paris and J. Řeháček (eds.), Quantum state estimation, Lecture Notes in Physics 649 (Springer, Heidelberg, 2004).

[2] I. D. Ivanovic, J. Phys. A 13, 3241 (1981); W. K. Wootters, Annals of Physics 176, 1 (1988).

[3] R. B. A. Adamson and A. M. Steinberg, Phys. Rev. Lett. 105, 030406 (2010).

[4] G. Lima et al, Optics Express 19, 3542 (2011).

[5] R. T. Thew, K. Nemoto, A. G. White, and W. J. Munro, Phys. Rev. A 66, 012303 (2002).

[6] I. Sainz, L. Roa, and A. B. Klimov, Phys. Rev. A 81, 052114 (2010).

[7] L. Roa, C. Hermann-Avigliano, R. Salazar, and A. B. Klimov, Phys. Rev. A 84, 014302 (2011).

[8] C. Paiva-Sánchez, E. Burgos-Inostroza, O. Jimenez, and A. Delgado, Phys. Rev. A 82, 032115 (2010).

[9] J. S. Lundeen and C. Bamber, Phys. Rev. Lett. 108, 070402 (2012).

[10] T. Cheon and S. Poghosyan, arXiv:1306.4767v1 (2013).

[11] J. S. Lundeen, B. Sutherland, A. Patel, C. Stewart, and C. Bamber, Nature 474, 188 (2011).

[12] L. M. Johansen, Phys. Rev. A 76, 012119 (2007).

[13] J. Z. Salvail, M. Agnew, A. S. Johnson, E. Bolduc, J. Leach, and R. W. Boyd, Nature Photonics 7, 316-321 (2013).

[14] S. Wu, Scientific Reports 3, 1193 (2013).

[15] Y. Aharonov, P. Bergmann, and J. Lebowitz, Phys. Rev. 134, B1410 (1964).

[16] B. Reznik and Y. Aharonov, Phys. Rev. A 52, 2538 (1995).

[17] Y. Aharonov, D. Z. Albert, and L. Vaidman, Phys. Rev. Lett. 60, 1351 (1988).

[18] A. G. Kofman, S. Ashhab, and F. Nori, Physics Reports 520, 43-133 (2010).

[19] Y. Aharonov, A. Botero, S. Popescu, B. Reznik, and J. Tollasksen, Phys. Lett. A 301, 130 (2002); S. Kocsis et al, Science 332, 1170 (2011); M. F. Pusey, Phys. Rev. Lett. 113, 200401 (2014).

[20] H. F. Hofmann, Phys. Rev. A 81, 012103 (2010).

[21] H. F. Hofmann, New J. Phys. 14, 043031 (2012).

[22] D. R. Solli, C. F. McCormick, R. Y. Chiao, S. Popescu, and J. M. Hickmann, Phys. Rev. Lett. 92, 043601 (2004); N. Bruner el al, Phys. Rev. Lett. 93, 203902 (2004).

[23] O. Hosten and P. Kwait, Science 319, 787 (2008); P. B. Dixon, D. J. Starling, A. N. Jordan, and J. C. Howell, Phys. Rev. Lett. 102, 173601 (2009); N. Brunner and C. Simon, Phys. Rev. Lett. 105, 010405 (2010); A. Feizpour, X. Xing, and A. M. Steinberg, Phys. Rev. Lett. 107, 133603 (2011).

[24] J. Wong, J. Math. Phys. 8, 2039 (1967); H. C. Baker, Phys. Rev. A 30, 773 (1984); T. Tanaka, J. Phys. A. Math. Gen. 39, 3715 (2006).

[25] I. Sainz, L. Roa, and A. B. Klimov, J. Math. Phys. 53, 052102 (2012). 